\title{
Childhood body mass index and development of eating disorder traits across adolescence
}

\author{
Camilla A. Wiklund ${ }^{1}$ | Ralf Kuja-Halkola ${ }^{1}$ (D) | Laura M. Thornton ${ }^{2}$ (i) | Katarina Bälter ${ }^{1,3}$ (D) | \\ Elisabeth Welch ${ }^{1,4}$ | Cynthia M. Bulik ${ }^{1,2,5}$ (D)
}

\footnotetext{
${ }^{1}$ Department of Medical Epidemiology and Biostatistics, Karolinska Institutet, Stockholm, Sweden

${ }^{2}$ Department of Psychiatry, University of North Carolina at Chapel Hill, Chapel Hill, North Carolina

${ }^{3}$ Division of Public Health Sciences, School of Health, Care and Social Welfare, Mälardalen University, Västerås, Sweden

${ }^{4}$ Stockholm Center for Eating Disorders, Stockholm, Sweden

${ }^{5}$ Department of Nutrition, University of North Carolina at Chapel Hill, Chapel Hill, North Carolina

Correspondence

Cynthia M. Bulik, PhD, Department of Medical Epidemiology and Biostatistics, Karolinska Institutet, Nobels väg 12A, Stockholm 171 77, Sweden.

Email: cbulik@med.unc.edu

Funding information

Swedish Research Council, Grant/Award Numbers: 2017-00641 and 538-2013-8864
}

\begin{abstract}
Objective: Understanding the role of premorbid body mass index (BMI) in the emergence of eating disorders may be key to identifying effective prevention strategies. We explore relations between BMI and eating disorders traits in young twins.

Method: The effect of BMI at age 9/12 and 15 on eating disorder traits measured using the Eating Disorders Inventory-2 (EDI) at ages 15 and 18 was examined using bivariate modelling in a longitudinal population sample of Swedish twins.

Results: The correlation between BMI and EDI within individuals was stable across all ages and remained significant after adjusting for later BMI. Bivariate analysis indicated significant positive genetic correlations between BMI ages 9/ 12 and 15 and subsequent EDI scores. The relationship remained significant for BMI age 9/12 and EDI age 15 in the adjusted model, indicating a longitudinal association.
\end{abstract}

Conclusion: Our results have implications for conceptualizing the interrelation of BMI and eating disorders across childhood and adolescence.

\section{KEYWORDS}

adolescence, BMI, childhood, eating disorders

\section{1 | INTRODUCTION}

Several lines of evidence underscore the importance of elucidating the etiological relation between eating disorder traits and body mass index (BMI). Results from a genome-wide association study (GWAS) revealed significant negative single nucleotide polymorphism (SNP)based genetic correlations between anorexia nervosa and BMI: Many of the same alleles influence these traits but act in opposing directions (Duncan et al., 2017). Additionally, data from the Avon Longitudinal Study of Parents and Children showed that early divergence from BMI-based growth trajectories predicted later onset of eating disorders-divergence below the norm was associated with later anorexia nervosa, and divergence above the norm was associated with later bulimia nervosa, purging disorder, and binge-eating disorder (Yilmaz, Gottfredson, Zerwas, Bulik, \& Micali, 2017). Low weight and dieting have also predicted subthreshold anorexia nervosa in an adult female population (Stice, Gau, Rohde, \& Shaw, 2017). However, other studies report that elevated premorbid BMI (Berkowitz et al., 2016) and larger childhood body size (Li, Eriksson, He, Hall, \& Czene, 2017) increased risk for anorexia nervosa. To further our understanding of the role that premorbid BMI plays in the emergence of eating disorders, we explored the 
developmental association between childhood BMI and later eating disorder traits in adolescence (as measured by the Eating Disorder Inventory-2, [EDI; Garner, 1991]) in a longitudinal twin sample. Twin designs allow us to explore not only the potential association but also the extent to which the association is due to common genetic and/or environmental factors. Understanding the nature of the relationship (e.g., a large genetic component could indicate common biological underpinnings, whereas a large environmental component could reflect a learned behavior or experienced condition) will inform future research and eventual prevention and treatment efforts.

Eating disorder traits are heritable and twin-based estimates of the heritability of EDI total and subscale scores range from $16 \%$ to $67 \%$ for females and $0 \%$ to $47 \%$ for males (Baker et al., 2009; Baker, Thornton, Bulik, Kendler, \& Lichtenstein, 2012; Keski-Rahkonen et al., 2005; Klump et al., 2012; Klump, McGue, \& Iacono, 2000; Rojo-Moreno et al., 2017); the broad ranges likely reflect sample differences including demographic factors such as age and country of origin. Genetic effects on EDI scores become more predominant across childhood in females, specifically in the transition from early to mid-adolescence (Klump, Burt, McGue, \& Iacono, 2007). This increase in genetic risk has been hypothesized to be moderated by pubertal development and increasing production of ovarian hormones such as estradiol in females (Klump, Keel, Sisk, \& Burt, 2010; Klump, McGue, \& Iacono, 2003).

SNP-based heritability estimates of BMI have been estimated to be $\sim 31 \%$ (Wills, Evans, \& Hopfer, 2017), with twin-based heritability estimates ranging from $47 \%$ to $90 \%$ (Elks et al., 2012). Moreover, GWAS of obesity has identified 112 BMI-associated loci (Akiyama et al., 2017). Like eating disorder traits, genetic factors influencing BMI become more prominent from middle childhood to adulthood (Elks et al., 2012).

Previous research investigating the relation between BMI and eating disorder traits has yielded inconsistent results. Both children with higher BMIs compared with peers and children who are overweight or obese are at greater risk of developing eating disorders and eating disorder symptoms (Berkowitz et al., 2016; Swenne, 2001; Tanofsky-Kraff et al., 2004; Wade et al., 2014). However, not all research concurs; other studies report no association with either premorbid underweight or overweight in relation to later eating disorders (Gardner, Stark, Friedman, \& Jackson, 2000; Nicholls \& Viner, 2009).

To clarify this relationship, we used longitudinal data from the Child and Adolescent Twin Study in Sweden (CATSS), which is part of the Swedish Twin Registry (Anckarsäter et al., 2012). Specifically, we explored the relation between BMI and the emergence of eating disorder traits as measured by three subscales of the EDI at ages 15 and 18, in both females and males. We hypothesized that childhood BMI would be significantly associated with later eating disorder symptoms. As previous research has found differences in genetic contribution between females and males during adolescence, we further hypothesized that estimates would differ by sex. By comparing the similarity between monozygotic (MZ) twin pairs with that of dizygotic (DZ) twin pairs, we estimated the genetic and environmental contributions to BMI and to the EDI variables. We conducted bivariate twin modelling, which expands the basic twin model and provides estimates of twin-based genetic and environmental correlations between BMI and EDI scores. These correlations estimate the extent to which genetic and environmental contributions to liability to the traits are shared and change during the transition from adolescence into young adulthood.

\section{2 | MATERIALS AND METHODS}

\section{1 | Sample}

CATSS is an ongoing, longitudinal, population-based study targeting all twins born in Sweden since July 1, 1992. Detailed information about recruitment and data collection has been described (Anckarsäter et al., 2012). In this present study, we used data from the parental telephone interview at age 9 or 12 and follow-up web-based questionnaires at ages 15 and 18. During the first 3 years of the study, parents of 12-year-old twins (born July 1992 to June 1995) and 9-year-old twins (born July 1995 and onwards) were invited to participate in parallel. After the initial 3 years, baseline recruitment was restricted to 9-year-old twins and the total baseline sample consists of approximately $80 \%$ 9-year olds. All participants gave informed consent before enrollment, parents gave informed consent before the telephone interview, and twins before the follow-up was conducted. We included all twins answering the EDI questions at age 15 or 18 and/or had recorded height and weight at any of the three ages. Response rate at baseline was approximately $80 \%$ and at follow-up approximately $60-70 \%$. CATSS has been approved by the Regional Ethical Review Board in Stockholm, Sweden.

\subsection{1 | Zygosity determination}

Zygosity was determined by a panel of 48 SNPs when DNA was available and otherwise by an algorithm based on five questions on twin similarity derived from pairs with known zygosity (Magnusson et al., 2013). 


\section{2 | Measures}

\subsection{1 | Eating Disorders Inventory-2}

Eating disorder traits are measured using the Drive for Thinness (DT), Bulimia (B), and Body Dissatisfaction (BD) subscales of the EDI (Garner, 1991). The DT measures excessive concern with dieting, preoccupation with weight, and fear of gaining weight; B measures binge eating and purging episodes; and BD measures dissatisfaction with one's physical appearance. We computed the three subscale scores ${ }^{1}$ and the total score (the sum of DT, B, and BD subscales). To capture the full range of eating disorder traits and allow for greater variance in the EDI subscales for this population-based sample, we used the full 6-point response scale for each item and then summed the respective items for each subscale. Thus, the highest possible scores were DT $=42, \mathrm{~B}=42$, and $\mathrm{BD}=48$. We investigated the association between BMI and total EDI score; EDI monotonically increased as BMI increased for all ages, not suggesting a J- or Ushaped relationship (data not shown).

\subsection{2 | Body mass index}

BMI (weight $[\mathrm{kg}] /$ height squared $\left[\mathrm{m}^{2}\right]$ ) was calculated for all participants based on parental-reported height and weight at age $9 / 12$ and by self-reported height and weight at ages 15 and 18 .

\subsection{Statistical analysis}

Means and standard deviations were calculated for the EDI total score and subscales and for BMI for the total sample and by sex.

We calculated within-trait correlations in $\mathrm{MZ}$ and $\mathrm{DZ}$ twins separately by sex and for opposite-sex DZ twins. We estimated the within-individual phenotypic correlations between BMI at ages 9/12 and 15 and EDI score at ages 15 and 18. We calculated intraclass correlations, that is, the correlation between BMI at one age in Twin 1 and EDI at a later age in Twin 2 and vice versa. A greater intraclass correlation in MZ than DZ twins suggests that the phenotypic correlation between the two traits can at least partly be explained by a common genetic component.

The analyses are based on standard assumptions of twin modelling. Namely, MZ twins share essentially $100 \%$ of their segregating alleles, whereas DZ twins share on average 50\%, and $\mathrm{MZ}$ and $\mathrm{DZ}$ twins share their

\footnotetext{
${ }^{1}$ In the data collection of CATSS at ages 15 and 18, one item from the $\mathrm{BD}$ subscale is missing (I like the shape of my buttocks); however, the reverse item (I think my buttocks are too large) is included.
}

environment to an equal extent. This enables decomposition of the variance in a phenotype and the covariance between phenotypes into additive genetic (A), shared environmental (C), and nonshared environmental components (including measurement error: E). Thus, MZ twins have a correlation of 1.0 for both $\mathrm{A}$ and $\mathrm{C}$ and no correlation for E. DZ twins have a correlation of 0.5 for A, 1.0 for C, and no correlation for E (Plomin, DeFries, Knopik, \& Neiderhiser, 2012).

\subsection{1 | Bivariate model}

We performed bivariate analyses to estimate the relative contribution of genetic and environmental factors to the covariance between BMI age 9/12 and EDI age 15 (Model 1), BMI age 9/12 and EDI age 18 (Model 2), and BMI age 15 and EDI age 18 (Model 3). We repeated this for all EDI subscales separately. We estimated the additive genetic, shared environmental, and nonshared environmental correlations. An additive genetic correlation is the proportion of variance that two traits share due to genetic factors; a value of 1.0 indicates $100 \%$ overlap between the additive genetic components in each of the phenotypes. Shared and nonshared environmental correlations are similarly defined. Additionally, bivariate heritability was estimated. Specifically, bivariate heritability reflects the fraction of the phenotypic correlation (the observable association between two traits) explained by genetic factors: It is a function of the heritabilities of the two traits and their genetic correlation. As previous reports of sex differences for both BMI and EDI exist, we allowed for quantitative and qualitative sex differences in our models. Quantitative differences occur when the influence of genetic or environmental factors on the phenotype differs in magnitude between sexes. This is modelled by allowing estimates of A, C, and E to be different in females and males. Qualitative differences arise when the genetic effect on a phenotype is different between sexes. We accounted for such differences by estimating the fraction of the A correlation between females and males in opposite-sexed pairs, compared with same-sexed pairs, varying from 0 to 1 (Neale, Roysamb, \& Jacobson, 2006). To not introduce bias and to be able to compare estimates between models and time points, we fit full bivariate models (Sullivan \& Eaves, 2002).

To control for the correlated nature of BMI at different ages, we repeated the analysis including BMI as a covariate in the models. In the models, we adjusted EDI for BMI measured contemporaneously, adjusting EDI age 15 for BMI age 15 in Model 1 and EDI age 18 for BMI age 18 in Models 2 and 3. 
All analyses were conducted using $\mathrm{R}$ version 3.2.2 software and statistical package OpenMx 2.7.11 (Neale et al., 2016).

\section{3 | RESULTS}

\section{1 | Descriptive statistics}

Table 1 presents means and standard deviations for BMI at all three ages for the total population and for females and males, separately. Table 1 also presents EDI total and subscale scores at ages 15 and 18 for the total population and by sex. As expected, females scored higher than males for total EDI and all subscales at both ages. All means increased significantly between ages 15 and 18, for both sexes (all $P$ values $<0.01$ ).

\section{2 | Twin correlations}

Twin correlations for BMI at all ages and for EDI ages 15 and 18 are presented in Table 2. MZ correlations for all measures are higher than DZ correlations suggesting genetic effects for both BMI and EDI scores. The phenotypic correlations were longitudinally stable across age and sex, ranging from 0.31 to 0.33 for females and 0.26 to 0.29 for males. Furthermore, the higher intraclass correlations for $\mathrm{MZ}$ compared with $\mathrm{DZ}$ twins suggests that the overlap between BMI and EDI is influenced by genetic factors.

\section{3 | Bivariate model-Unadjusted for BMI}

Results from the full bivariate models including BMI and EDI total score at different ages are presented in Table 3A-C. For BMI, the majority of the variance was explained by additive genetic factors for females and males at both age 9/12 and 15. For both sexes, the
TABLE 2 Twin, intraclass, and phenotypic correlations for BMI and EDI measures

\begin{tabular}{lccccc} 
& MZf & DZf & MZm & DZm & DZos \\
$N$ & 3,163 & 3,375 & 3,064 & 3,725 & 7,089 \\
Twin correlations & & & & & \\
BMI 9/12 & 0.87 & 0.53 & 0.88 & 0.52 & 0.49 \\
BMI 15 & 0.82 & 0.45 & 0.82 & 0.38 & 0.22 \\
BMI 18 & 0.74 & 0.53 & 0.76 & 0.41 & 0.28 \\
EDI 15 & 0.65 & 0.33 & 0.54 & 0.19 & 0.17 \\
EDI 18 & 0.63 & 0.35 & 0.48 & 0.17 & 0.22 \\
\hline Intraclass correlations & & & & & \\
BMI 9/12-EDI 15 & 0.32 & 0.11 & 0.27 & -0.01 & 0.09 \\
BMI 9/12-EDI 18 & 0.31 & 0.18 & 0.22 & 0.08 & 0.08 \\
BMI 15-EDI 18 & 0.33 & 0.20 & 0.20 & 0.07 & 0.09 \\
\hline
\end{tabular}

Note. BMI: body mass index; EDI: Eating Disorders Inventory-2; MZf: monozygotic female twin pairs; DZf: dizygotic female twin pairs; MZm: monozygotic male twin pairs; DZm: dizygotic male twin pairs; DZos: dizygotic opposite sex twin pairs.

proportion of variance explained by additive genetic factors increased between ages $9 / 12$ and 15 and the variance explained by shared environmental factors decreased. The variance explained by the nonshared environmental component increased slightly for both females and males between ages $9 / 12$ and 15 .

For the EDI total score, estimates of variance explained by additive genetic factors were higher for females than for males at both ages 9/12 and 15. At age 15 (Table 3A), the contribution of additive genetic factors and nonshared environmental factors were estimated to be $66 \%$ and $34 \%$ in females and 57\% and 43\% in males. At age 18 (Tables 3B and $3 \mathrm{C}$ ), the contribution of additive genetic factors decreased for both females and males compared with age 15; however, the confidence intervals overlapped. The shared environment contribution was estimated to be near $0 \%$ in all models at both age 15 and 18.

TABLE 1 Means $(S D)$ for BMI and EDI full score and subscale scores for the total population and by sex

\begin{tabular}{|c|c|c|c|c|c|c|c|c|c|}
\hline & \multicolumn{3}{|l|}{ 9/12 years } & \multicolumn{3}{|l|}{15 years } & \multicolumn{3}{|l|}{18 years } \\
\hline$N$ & 26,400 & 12,904 & 13,496 & 9,372 & 5,148 & 4,224 & 7,230 & 4,157 & 3,073 \\
\hline BMI & $16.97(2.52)$ & $16.95(2.60)$ & $16.98(2.43)$ & $20.51(2.76)$ & $20.46(2.72)$ & $20.58(2.72)$ & $21.95(3.15)$ & $21.63(3.16)$ & $22.38(3.10)$ \\
\hline EDI total & - & - & - & $46.74(17.21)$ & $53.27(18.19)$ & 38.72 (11.69) & $51.89(19.16)$ & $58.90(19.72)$ & $42.20(13.24)$ \\
\hline BD & - & - & - & 21.00 (9.09) & 24.38 (9.10) & $16.81(7.11)$ & 23.16 (9.07) & $26.34(8.93)$ & 18.77 (7.24) \\
\hline B & - & - & - & $10.81(4.09)$ & $11.41(4.40)$ & $10.08(3.53)$ & $12.44(5.19)$ & $13.33(5.67)$ & $11.20(4.14)$ \\
\hline
\end{tabular}

Note. BMI: body mass index $\left(\mathrm{kg} / \mathrm{m}^{2}\right)$; EDI: Eating Disorders Inventory-2; DT: drive for thinness; BD: body dissatisfaction; B: bulimia. 

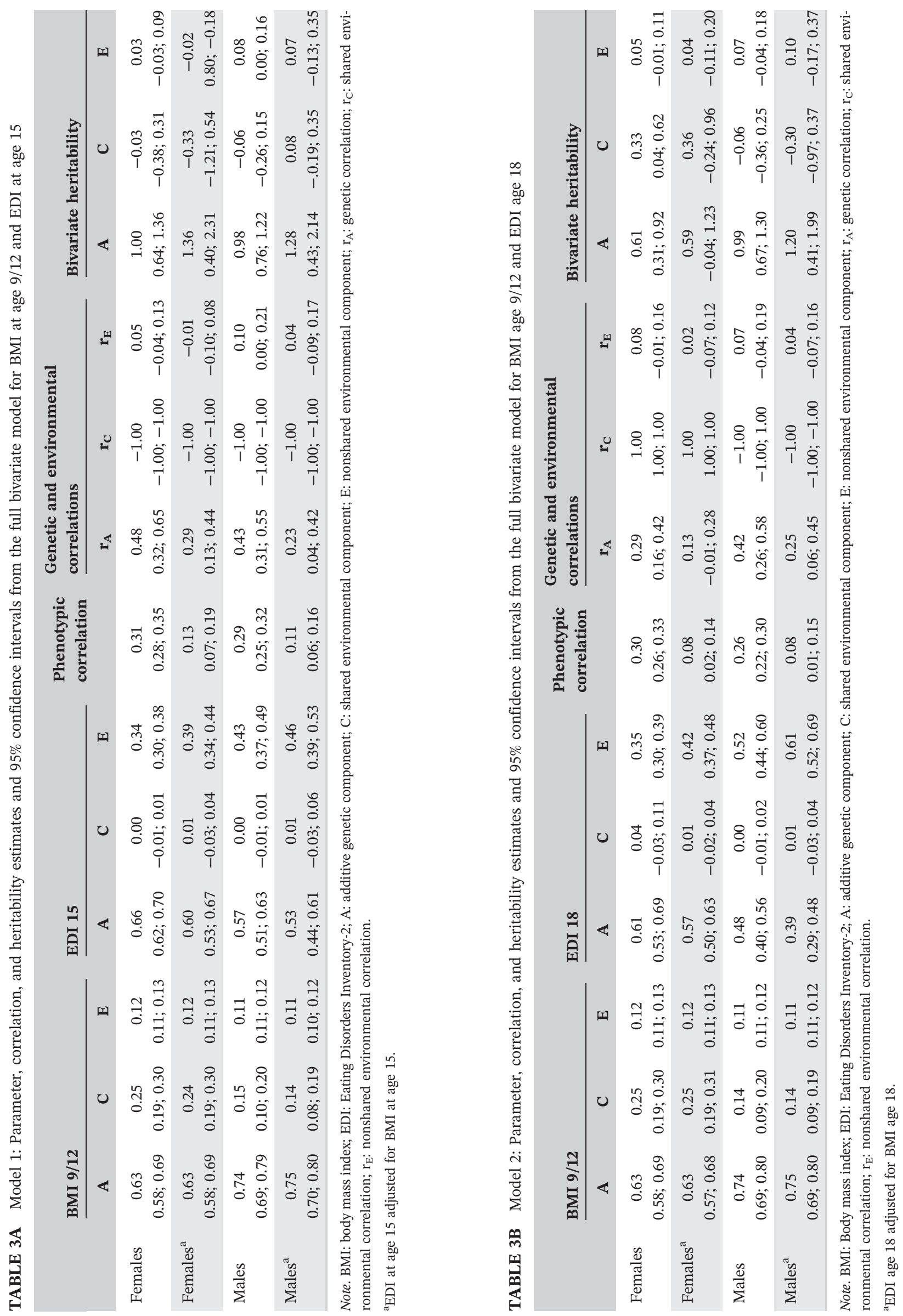


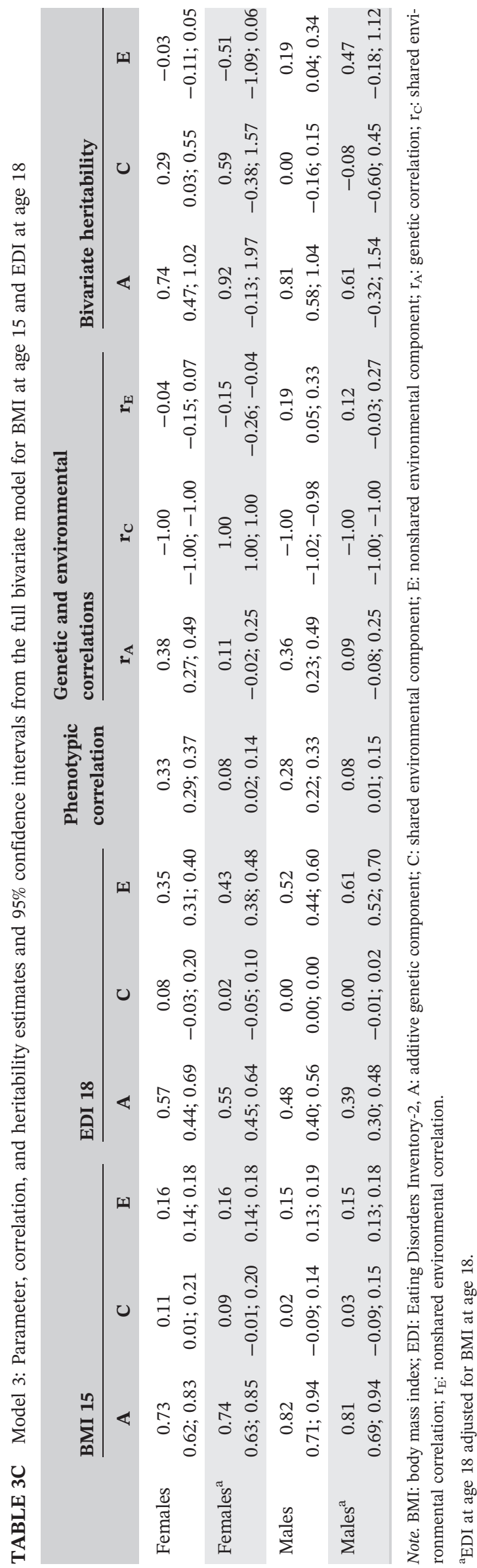

The genetic correlation between BMI age 9/12 and EDI at age 15 in Model 1 was estimated to be 0.48 for females and 0.43 for males (Table $3 \mathrm{~A}$ ), and the bivariate heritability was estimated to be 1.00 for females and 0.98 for males in Model 1. These results indicate that there is considerable but not complete overlap in genetic factors that contribute to the liability to these two traits and that the genetic factors almost entirely account for the observed phenotypic correlation. In males, the genetic correlations between BMI age 9/12 and EDI age 15 in Model 1 and between BMI age 9/12 and EDI age 18 in Model 2 (Table 3B) were similar. For females in Model 2, the genetic correlation was lower between BMI age 9/ 12 and EDI age 18 than it was in Model 1; however, confidence intervals overlapped. In Model 2, the bivariate heritability was estimated to be 0.99 for males and 0.61 for females. For females, the overlap of genetic factors between BMI age 9/12 and EDI age 18 was than less than that observed in Model 1. Both genetic and environmental factors that are shared by BMI age 9/12 and EDI age 18 contribute to the phenotypic correlation. The genetic correlation between BMI age 15 and EDI age 18 in Model 3 (Table 3C) was similar between females and males, estimated to be 0.38 and 0.36 , respectively. In this model, the bivariate heritability was lower for males but higher for females, compared with Model 2; however, all confidence intervals were overlapping. The results for Model 3 indicate that BMI age 15 and EDI age 18 share considerable genetic factors in both males and females and the shared genetic factors account for most of the association between these two traits.

\section{4 | Bivariate model-Adjusted for BMI}

Tables 3A-3C also present the estimates for the models when EDI is adjusted for contemporaneous BMI. In all models, adjusting for BMI led to reductions in estimates of both phenotypic correlations and genetic correlations. The estimates for the phenotypic correlations between BMI and EDI were, however, still positive and significant in all models. Thus, in Model 1 (Table 3A), some of the observed correlation between BMI age 9/12 and EDI age 15 was explained by the association between BMI age 15 and EDI age 15 and by the association between BMI at the two ages. However, a significant genetic correlation between the two traits remained in this model. Note that in the adjusted model, the bivariate heritability exceeded 1 . This is because the estimates are expressed in proportions and sum to 1: Since the bivariate shared environmental effect was negatively associated with BMI, the bivariate heritability is outside of the range 0 and 1. In this case, the bivariate heritability should be interpreted as explaining a very large proportion of the 
phenotypic correlation. In Model 2 (Table 3B), including BMI age 9/12 and EDI age 18 and adjusting for BMI age 18 , the pattern was similar; however, the genetic correlation in the adjusted model was only still significant in males. In the third model (Table 3C), the genetic correlation decreased to 0.08 for both females and males and became nonsignificant.

The results for the DT and the BD subscales follow a similar pattern to the EDI total score. For the B subscale, the phenotypic correlations with BMI were all very low. Results for all three subscales are presented in Tables S1-S3.

\section{4 | DISCUSSION}

In this population-based longitudinal twin study, we observed significant positive phenotypic correlations between BMI and eating disorder traits measured by the EDI at all ages. When controlling for later BMI, the phenotypic correlations, although slightly lower, remained significant in all models. We also found significant positive genetic correlations between BMI at younger ages and EDI scores in later adolescence. The genetic correlation between BMI age 9/12 and EDI age 15 remained significant after controlling for BMI at age 15. These results add to our understanding of common etiological pathways between BMI and eating disorders traits by highlighting a common genetic effect (i.e., significant genetic correlations) between these two traits in a longitudinal model, which suggests that some of the same genetic factors play a causal role for both BMI and eating disorder traits. That is, elevated, premorbid BMI may be a risk factor for later disordered eating.

Klump et al. investigated the overlap between BMI in childhood and eating disorder traits in adolescence, on a smaller, female-only, twin sample (Klump, McGue, \& Iacono, 2000). They reported a positive association between higher BMI and higher EDI scores. Our study supports and extends those findings in two ways. First, we report on a much larger sample that is both population based and longitudinal, allowing us to detect associations that previous research has not been adequately powered to detect. Longitudinal measures increase the ability to isolate effects that BMI has on eating disorder traits in different phases of the transition from childhood through adolescence. Second, our sample contains both females and males, enabling a deeper understanding of sex differences in the developmental course of eating disorders traits. Previous research suggests that there are differences in genetic and environmental contributions for eating disorders traits between females and males (Baker et al., 2009; Klump et al., 2012; Rojo-Moreno et al., 2017).
This study clarifies those findings by elucidating the role of childhood and adolescent BMI in the observed associations.

The longitudinal design allowed us to control for BMI measured contemporaneously with EDI, ensuring that we are observing correlations between earlier BMI and later eating disorder traits, not simply the effect of the high correlation of BMI with itself over time. In the typical developmental course of female eating disorders, symptoms usually arise in adolescence (Herpertz-Dahlmann et al., 2015; Micali et al., 2015; Micali, Ploubidis, De Stavola, Simonoff, \& Treasure, 2014) and during the same period, most adolescents experience physical and emotional changes (Chulani \& Gordon, 2014). Previous twin studies have explored, for example, timing of menstruation, pubertal development, and female hormonal levels in relation to eating disorder traits (Baker, Thornton, Bulik, Kendler, \& Lichtenstein, 2012; Klump, 2013; Klump et al., 2012; Klump, Keel, Sisk, \& Burt, 2010). Our results reveal that even after adjusting for BMI, phenotypic correlations in all models remain significant, indicating a longitudinal, stable association between BMI and eating disorder traits. Previous studies that did not account for the effect of BMI may have overestimated the magnitude of the observed associations between puberty and eating disorder traits.

The subscales DT and BD follow the pattern of results for EDI total scale in both sexes. However, in the B subscale, the average score was lower than the two other scales and the phenotypic correlations with BMI were close to zero. This is not surprising; in a population sample, few individuals are expected to endorse bulimic behaviours. Other studies in similar settings have found comparable results (Baker et al., 2009).

These results are also consistent with the recent molecular genetic studies evaluating the association among eating disorders and BMI on a genetic level (Duncan et al., 2017). Also, the studies reporting that high childhood BMI is associated with risk for eating disorders, including anorexia nervosa, bulimia nervosa, and binge-eating disorder, map well onto our findings (Berkowitz et al., 2016; Swenne, 2001; Yilmaz, Gottfredson, Zerwas, Bulik, \& Micali, 2017). Although we did not examine clinically diagnosed disorders, our results nonetheless contribute to increased knowledge as these differences have been observed between BMI and threshold eating disorders.

It should be noted that these analyses do not identify specific liability factors that these traits have in common. Thus, we are unable to determine who may be most vulnerable to the development of disordered eating. However, the substantial genetic correlations and the high bivariate heritabilites observed encourage next scientific 
steps to identify specific genetic regions and biological pathways that are shared between the traits during the developmental period of adolescence. The increasing specificity that such approaches afford, together with BMI trends, has the potential to assist with prediction and early intervention to mitigate adverse outcomes.

\section{1 | Limitations}

Limitations should be considered. Previous research on the EDI has found individuals with bulimia nervosa score higher than individuals with anorexia nervosa. Those with anorexia nervosa may underestimate or be unable to acknowledge symptoms, experiencing them as more ego syntonic (Nevonen \& Broberg, 2001). This would mean that we preferentially capture certain aspects of eating disorder traits.

Further, the missing item from the total score and BD subscale could render our means slightly lower than other studies. Nevertheless, the items in the scale are highly correlated; we therefore expect results to be similar to those from BD subscales without the missing item. The total and subscale scores agree well with previously seen numbers in Sweden, for both females and males (Baker et al., 2009).

The BMI scores are based on parental-reported or self-reported data. However, studies have found that self-reported height and weight show high validity compared with objectively measured estimates for Swedish adolescents (Ekström, Kull, Nilsson, \& Bergström, 2015).

Finally, we evaluated eating disorder traits assessed by the EDI, rather than clinical diagnosis of eating disorders-which have low population prevalence. Therefore, how our results generalize to individuals with threshold eating disorders is unknown.

In conclusion, we studied a large, longitudinal, population-based twin cohort to explore the relationship between child and adolescent BMI and later eating disorder traits. The results revealed significant, longitudinal phenotypic correlations in all models, which remained after adjusting for contemporaneously measured BMI. We observed significant genetic correlations between BMI and EDI total score at all ages. These results inform our conceptualization of the relationship between BMI and eating disorders across childhood and adolescence. Future studies should explicate specific commonalities in genetic contributions that influence both BMI and eating disorder traits during the developmental period of adolescence using both twin and molecular genetic designs to identify specific biological liability factors.

\section{ACKNOWLEDGEMENTS}

Dr Bulik acknowledges funding from the Swedish Research Council (VR Dnr: 538-2013-8864). We acknowledge The Swedish Twin Registry for access to data. The Swedish Twin Registry is managed by Karolinska Institutet and receives funding through the Swedish Research Council (VR Dnr: 2017-00641).

\section{CONFLICT OF INTEREST}

Dr Bulik is a grant recipient from Shire and has served on their Scientific Advisory Board.

\section{ORCID}

Ralf Kuja-Halkola (D) http://orcid.org/0000-0002-3765-2067

Laura M. Thornton (D) http://orcid.org/0000-0001-9384-7988

Katarina Bälter (D) http://orcid.org/0000-0003-2046-5641

Cynthia M. Bulik (D) http://orcid.org/0000-0001-7772-3264

\section{REFERENCES}

Akiyama, M., Okada, Y., Kanai, M., Takahashi, A., Momozawa, Y., Ikeda, M., ... Kamatani, Y. (2017). Genome-wide association study identifies 112 new loci for body mass index in the Japanese population. Nature Genetics, 49, 1458-1467. https://doi. org/10.1038/ng.3951

Anckarsäter, H., Lundström, S., Kollberg, L., Kerekes, N., Palm, C., Carlström, E., ... Lichtenstein, P. (2012). The child and adolescent twin study in Sweden (CATSS). Twin Research and Human Genetics, 14, 495-508. https://doi.org/10.1375/twin.14.6.495

Baker, J. H., Maes, H. H., Lissner, L., Aggen, S. H., Lichtenstein, P., \& Kendler, K. S. (2009). Genetic risk factors for disordered eating in adolescent males and females. Journal of Abnormal Psychology, 118, 576-586. https://doi.org/10.1037/a0016314

Baker, J. H., Thornton, L. M., Bulik, C. M., Kendler, K. S., \& Lichtenstein, P. (2012). Shared genetic effects between age at menarche and disordered eating. Journal of Adolescent Health, 51, 491-496. https://doi.org/10.1016/j.jadohealth.2012.02.013

Berkowitz, S. A., Witt, A. A., Gillberg, C., Råstam, M., Wentz, E., \& Lowe, M. R. (2016). Childhood body mass index in adolescentonset anorexia nervosa. International Journal of Eating Disorders, 49, 1002-1009. https://doi.org/10.1002/eat.22584

Chulani, V. L., \& Gordon, L. P. (2014). Adolescent growth and development. Primary Care, 41, 465-487. https://doi.org/ 10.1016/j.pop.2014.05.002

Duncan, L., Yilmaz, Z., Gaspar, H., Walters, R., Goldstein, J., Anttila, V., ... Bulik, C. M. (2017). Significant locus and metabolic genetic correlations revealed in genome-wide association study of anorexia nervosa. The American Journal of Psychiatry, 174, 850-858. https://doi.org/10.1176/appi.ajp.2017.16121402

Ekström, S., Kull, I., Nilsson, S., \& Bergström, A. (2015). Web-based self-reported height, weight, and body mass index among 
Swedish adolescents: A validation study. Journal of Medical Internet Research, 17, e73. https://doi.org/10.2196/jmir.3947

Elks, C. E., den Hoed, M., Zhao, J. H., Sharp, S. J., Wareham, N. J., Loos, R. J., \& Ong, K. K. (2012). Variability in the heritability of body mass index: A systematic review and meta-regression. Frontiers in Endocrinology, 3, 29. https://doi.org/10.3389/ fendo.2012.00029

Gardner, R. M., Stark, K., Friedman, B. N., \& Jackson, N. A. (2000). Predictors of eating disorder scores in children ages 6 through 14: A longitudinal study. Journal of Psychosomatic Research, 49, 199-205.

Garner, D. M. (1991). Eating Disorder Inventory-2: Professional manual. Odessa, FL: Psycological Assessment Resourses Inc.

Herpertz-Dahlmann, B., Dempfle, A., Konrad, K., Klasen, F., Ravens-Sieberer, U., \& group, Bella study (2015). Eating disorder symptoms do not just disappear: The implications of adolescent eating-disordered behaviour for body weight and mental health in young adulthood. European Child \& Adolescent Psychiatry, 24, 675-684. https://doi.org/10.1007/s00787-0140610-3

Keski-Rahkonen, A., Bulik, C. M., Neale, B. M., Rose, R. J., Rissanen, A., \& Kaprio, J. (2005). Body dissatisfaction and drive for thinness in young adult twins. International Journal of Eating Disorders, 37, 188-199. https://doi.org/10.1002/ eat.20138

Klump, K. L. (2013). Puberty as a critical risk period for eating disorders: A review of human and animal studies. Hormones and Behavior, 64, 399-410. https://doi.org/10.1016/j. yhbeh.2013.02.019

Klump, K. L., Burt, S. A., McGue, M., \& Iacono, W. G. (2007). Changes in genetic and environmental influences on disordered eating across adolescence: A longitudinal twin study. Archives of General Psychiatry, 64, 1409-1415. https://doi.org/10.1001/ archpsyc.64.12.1409

Klump, K. L., Culbert, K. M., Slane, J. D., Burt, S. A., Sisk, C. L., \& Nigg, J. T. (2012). The effects of puberty on genetic risk for disordered eating: Evidence for a sex difference. Psychological Medicine, 42, 627-637. https://doi.org/10.1017/ S0033291711001541

Klump, K. L., Keel, P. K., Sisk, C., \& Burt, S. A. (2010). Preliminary evidence that estradiol moderates genetic influences on disordered eating attitudes and behaviors during puberty. Psychological Medicine, 40, 1745-1753. https://doi.org/10.1017/ S0033291709992236

Klump, K. L., McGue, M., \& Iacono, W. G. (2000). Age differences in genetic and environmental influences on eating attitudes and behaviors in preadolescent and adolescent female twins. Journal of Abnormal Psychology, 109, 239-251. https://doi.org/ 10.1037//0021-843X.109.2.239

Klump, K. L., McGue, M., \& Iacono, W. G. (2003). Differential heritability of eating attitudes and behaviors in prepubertal versus pubertal twins. International Journal of Eating Disorders, 33, 287-292. https://doi.org/10.1002/eat.10151

Li, J., Eriksson, M., He, W., Hall, P., \& Czene, K. (2017). Associations between childhood body size and seventeen adverse outcomes: Analysis of 65,057 European women. Scientific Reports, 7, 16917. https://doi.org/10.1038/s41598-017-17258-5

Magnusson, P. K., Almqvist, C., Rahman, I., Ganna, A., Viktorin, A., Walum, H., ... Lichtenstein, P. (2013). The Swedish Twin Registry: Establishment of a biobank and other recent developments. Twin Research and Human Genetics, 16, 317-329. https://doi.org/10.1017/thg.2012.104

Micali, N., De Stavola, B., Ploubidis, G., Simonoff, E., Treasure, J., \& Field, A. E. (2015). Adolescent eating disorder behaviours and cognitions: Gender-specific effects of child, maternal and family risk factors. The British Journal of Psychiatry, 207, 320-327. https://doi.org/10.1192/bjp.bp.114.152371

Micali, N., Ploubidis, G., De Stavola, B., Simonoff, E., \& Treasure, J. (2014). Frequency and patterns of eating disorder symptoms in early adolescence. Journal of Adolescent Health, 54, 574-581. https://doi.org/10.1016/j.jadohealth.2013.10.200

Neale, M. C., Hunter, M. D., Pritikin, J. N., Zahery, M., Brick, T. R., Kickpatrick, R. M., ... Boker, S. M. (2016). OpenMx 2.0: Extended structural equation and statistical modeling. Psychometrika, 80, 535-549. https://doi.org/10.1007/s11336-014-9435-8

Neale, M. C., Roysamb, E., \& Jacobson, K. (2006). Multivariate genetic analysis of sex limitation and $\mathrm{G} \times \mathrm{E}$ interaction. Twin Research and Human Genetics, 9, 481-489. https://doi.org/ 10.1375/183242706778024937

Nevonen, L., \& Broberg, A. G. (2001). Validating the Eating Disorder Inventory-2 (EDI-2) in Sweden. Eating and Weight Disorders, 6, 59-67. https://doi.org/10.1007/BF03339754

Nicholls, D. E., \& Viner, R. M. (2009). Childhood risk factors for lifetime anorexia nervosa by age 30 years in a national birth cohort. Journal of the American Academy of Child \& Adolescent Psychiatry, 48, 791-799. https://doi.org/10.1097/ CHI.0b013e3181ab8b75

Plomin, R., DeFries, J. C., Knopik, V. S., \& Neiderhiser, J. M. (2012). Behavior Genetics. New York, NY: Worth Publishers.

Rojo-Moreno, L., Iranzo-Tatay, C., Gimeno-Clemente, N., BarberaFons, M. A., Rojo-Bofill, L. M., \& Livianos-Aldana, L. (2017). Genetic and environmental influences on psychological traits and eating attitudes in a sample of Spanish schoolchildren. Revista de Psiquiatria y Salud Mental, 10, 134-142. https://doi. org/10.1016/j.rpsm.2015.05.003

Stice, E., Gau, J. M., Rohde, P., \& Shaw, H. (2017). Risk factors that predict future onset of each DSM-5 eating disorder: Predictive specificity in high-risk adolescent females. Journal of Abnormal Psychology, 126, 38-51. https://doi.org/10.1037/abn0000219

Sullivan, P. F., \& Eaves, L. J. (2002). Evaluation of analyses of univariate discrete twin data. Behavior Genetics, 32, 221-227. https://doi.org/10.1023/A:1016025229858

Swenne, I. (2001). Changes in body weight and body mass index (BMI) in teenage girls prior to the onset and diagnosis of an eating disorder. Acta Paediatrica, 90, 677-681.

Tanofsky-Kraff, M., Yanovski, S. Z., Wilfley, D. E., Marmarosh, C., Morgan, C. M., \& Yanovski, J. A. (2004). Eating-disordered behaviors, body fat, and psychopathology in overweight and normal-weight children. Journal of Consulting and Clinical Psychology, 72, 53-61. https://doi.org/10.1037/ 0022-006X.72.1.53 
Wade, K. H., Skugarevsky, O., Kramer, M. S., Patel, R., Bogdanovich, N., Vilchuck, K., ... Martin, R. M. (2014). Prospective associations of parental smoking, alcohol use, marital status, maternal satisfaction, and parental and childhood body mass index at 6.5 years with later problematic eating attitudes. Nutrition \& Diabetes, 4, e100. https://doi.org/10.1038/nutd.2013.40

Wills, A. G., Evans, L. M., \& Hopfer, C. (2017). Phenotypic and genetic relationship between BMI and drinking in a sample of UK adults. Behavior Genetics, 47, 1-8. https://doi.org/10.1007/ s10519-017-9838-2

Yilmaz, Z., Gottfredson, N. C., Zerwas, S. C., Bulik, C. M., \& Micali, N. (2017). Developmental premorbid body mass index trajectories of adolescents with eating disorders in a longitudinal population cohort. Manuscript under review.

\section{SUPPORTING INFORMATION}

Additional supporting information may be found online in the Supporting Information section at the end of the article.

How to cite this article: Wiklund CA, KujaHalkola R, Thornton LM, Bälter K, Welch E, Bulik CM. Childhood body mass index and development of eating disorder traits across adolescence. Eur Eat Disorders Rev. 2018;1-10. https://doi.org/10.1002/ erv. 2612 